\title{
Holistic optimization of train traffic by integration of automatic train operation with centralized train management
}

\author{
X. Rao ${ }^{1}$, M. Montigel ${ }^{1} \&$ U. Weidmann ${ }^{2}$ \\ ${ }^{I}$ Systransis Ltd., Switzerland \\ ${ }^{2}$ Institute for Transport Planning and Systems, ETH, Switzerland
}

\begin{abstract}
Nowadays, railways are confronted with numerous pressing problems, including capacity optimization, energy conservation, cost reduction and improving customer satisfaction. While the traditional railway is a very safe means of transportation, it still cannot meet all these requirements. Hence there are high interests in two available systems to overcome these challenges: Traffic Management System (TMS) and the optimized Automatic Train Operation (ATO). TMS is an efficient solution, which centralizes data to the railway infrastructure manager, while optimized ATO is an on-board approach available to minimize the loss of efficiency caused by manual operation. Until now, there are a lot of research studies about TMS and ATO as separate subjects. However, there is only poor research, which aims at closely coordinating the optimization strategies of TMS and ATO. This is because TMS is limited in the network scheduling optimization, while optimized ATO is centred on train behaviour optimization. Therefore this article presents the key idea of this research is to bridge the gap between TMS and ATO, to make progress towards combining the knowledge from both the infrastructure side and the train side. The primary objective is to bring an added value for achieving higher capacity, less energy consumption and higher operation quality by integration of Automatic Train Operation with centralized Train Management. In addition, an optimized ATO approach is introduced by integration of predictive fuzzy control with analytical hierarchy process. If this research has a positive response, it could bring about a new
\end{abstract}


method for railway optimization. The potential improved railway performance in addition to cost reduction could be highly beneficial to society.

Keywords: optimized automatic train operation, traffic management system, integration, multi-objectives optimization, predictive fuzzy control, analytical hierarchy process.

\section{Introduction}

With continuous increase in the need for transportation, more and more passengers and cargo have to be carried by rail. Consequently, railway operators are facing a range of challenges to improve existing railway system and to reduce cost. Such initiatives aim at a high quality traffic system with increase of capacity and efficiency on the railway network, at a more eco-friendly system with energy cost reduction, at higher customer-satisfaction thanks to better punctuality, reliability and information, at a flexible traffic management system to deal with deviations, incidents and breakdowns, at a highly automated system with efficient operation, and at a seamless integration of all the heterogeneous systems. As purpose of the conventional railway signalling system is exclusively to provide the safe train operation, it can meet the further requirements mentioned before to a very limited extent only. Hence Traffic Management System (TMS) and optimized Automatic Train Operation (ATO) are regarded as the promising solutions to meet these challenges. TMS comprises all functions and means necessary for enabling trains to run efficiently on the railway infrastructure [1]. Optimized ATO is an on-board concept for all phases of train operation, from acceleration to precise stopping, which implements train level optimization to help the train operators realize automation and exact operation. The following chapter will give an introduction of current research state of TMS and ATO separately.

\section{State of the art}

\subsection{Traffic Management System (TMS)}

Since the 1970s, TMS has been introduced at the core of railway systems for purposes such as automating route control, making efficient dispatch operations as well as passenger service [2]. Over the years, due to the growing transport demands, a more functional and integrated TMS promises advanced performances. A general function of the TMS is described in Figure 1 which builds a single centralized platform to maximize operational efficiency. It includes operation management, infrastructure management, traffic planning, passenger information system, staff information system, driver operation support system, maintenance management, training system, and so on [3].

There are several scientific projects carried out in different countries based on this general TMS function structure. For example, in Japan, the Hitachi TMS is best characterized by improving the dispatch operations in multiple lines [2]. In Europe, the Telvent TMS of Spain integrates all rail systems and subsystem into 


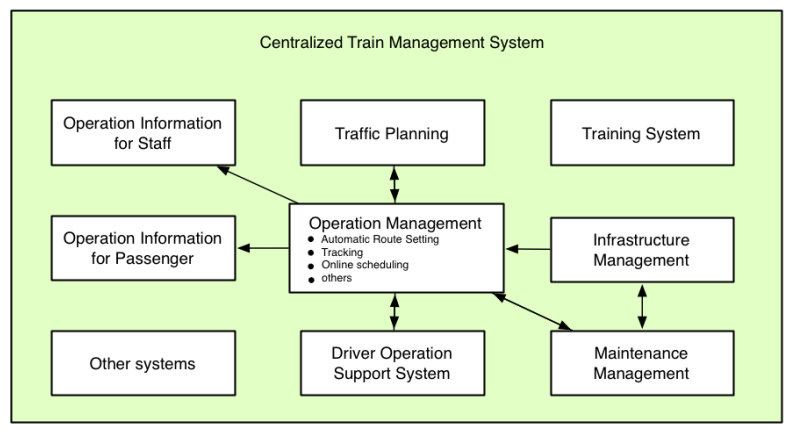

Figure 1: Overview of traffic management system [3].

a single platform that maximizes operational efficiency, which can be configured to whatever the size of the rail network [4]. While the Thales TMS, namely Advanced Railway Automation Management and Information System (ARAMIS), has been utilised for many projects. Its integrated, modular, freely scalable architecture facilitates to maintain, extend and run the system very costefficient [5]. Another state-of-the-art TMS system is applied in the new Lötschberg Base Tunnel in Switzerland, which is partially single-track. It is equipped with its own operations control system, called Automatic Function (AF) developed by Systransis Ltd. The practical experience gained on this line with optimized rescheduling in addition to reducing energy consumption proves to be excellent. AF enables Lötschberg Base Tunnel to realize high capacity as maximum 142 trains per day, and it saves about CHF 40 million in energy cost for an entire year $[6,7]$. AF has developed the first operational mainline conflict resolution and speed advisory system in the world. Lüthi [8] shows the key factor to achieve such improvement in the following Figure 2, that AF system has proposed a new rescheduling framework based on the superimposition of two general closed control loops: the outer feedback control loop supervises the train traffic and infrastructure state, detects deviations and conflicts, develops a new production plan based on optimization objectives, and transmits the new production. The inner feedback control loop on the lowest layer is responsible for executing the production plan, which is achieved by designing the supporting tools to send advisory speed profile to the driver. Therefore, the AF system enhances the TMS function of rescheduling as well as driver operation support.

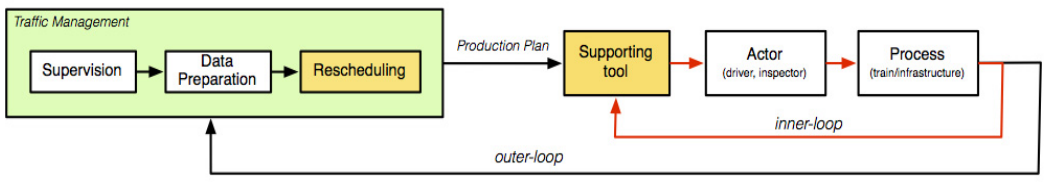

Figure 2: $\quad$ General TMS rescheduling control loops framework [8]. 
All in all, there are numerous promising approaches of TMS, but all these methods take train behaviours as given.

\subsection{Automatic Train Operation (ATO)}

Another optimization idea being undertaken is ATO. The main functions of ATO are automatic train speed control (propulsion, braking, cruising and coasting), parking, and door control. In view of the predefined control loop, ATO is actually an optimization in the inner feedback control loop. Figure 3 depicts the general structure of ATO, which makes the optimization calculation and implementation both in the inner-control loop. From this point of view, compared to TMS, ATO is a train level optimization.

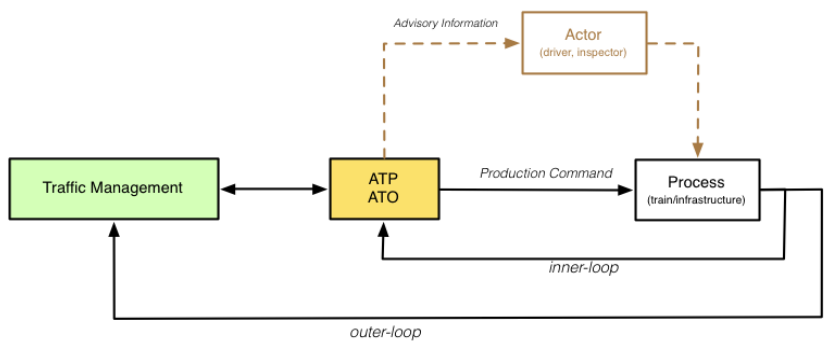

Figure 3: ATO control loop.

ATO is built on top of an Automatic Train Protection (ATP) system to provide the high integrity SIL-4 (Safety Integrity Level 4) safety functionality, which controls speeding trains and ensures safety. Consequently, the ATO system is not considered safety critical; it could be seen as a complementary or as an addition to ATP, building an integrated system [9].

Conventional ATO follows an optimal speed profile taking into account speed restrictions, timetable, and so on. Optimized ATO, as one advanced form of ATO, does not only aim at automatically controlling the train to follow the speed profile, but also in optimizing the train trajectory with multiple optimization goals, such as capacity increasing, comfort improving, energy saving, accurate stopping, and so on [10].

Until now, optimized ATO is widely used in metros, but seldom in mainline [11]. One successful application in the high-speed line is the Shinkansen train from Morioka to Hachinohe in Japan, which shows a good accuracy to follow the target speed and improvement in saving energy consumption [12]. It should be pointed out that ATO does not automatically imply unattended operation (UTO). In contrary, ATO is specified with several automation levels, and today's most common applications are in the basic level, in which the driver still remains on the train.

\subsection{Lessons learnt}

Lessons learnt from the two methods are that TMS is best suited for rescheduling, but with shortages in train-level optimization. Contrary to TMS, 
ATO is strong in getting real-time information of train specifics, which enables ATO to perform accurate execution of given traffic directives, locomotive calculation, and multi-objectives control. However, the resulting lack of knowledge about other trains' states and infrastructure information is perceived as the primary weakness of ATO to prevent conflicts efficiently. In addition, there is a lack of ATO experience on main line railways, especially in the mixed traffic lines, which might contain "ATP+ATO" equipped trains and "ATP only" equipped trains and other "non-ATP" equipped trains.

\section{Methodology used}

\subsection{Integration of TMS and ATO}

To tackle these challenges, the main idea of this research is to join together TMS and ATO by combining efforts from both infrastructure and trains, thereby making progress towards higher performance of the railway system at lower cost. For this aim, Figure 4 illustrates a clear picture of the newly proposed framework. The most innovative technical feature is the red dashed integration box, which is allocated at the interface between infrastructure and train, and which symbolizes the close integration of optimization strategies between infrastructure and train. The inputs of ATO are the production plan from TMS and the feedback data from the train. The outputs of ATO are either the direct commands to the train system, or the advisory information to the driver, and feedbacks to the TMS as well. With this integration method, it aims at optimizing behaviours of the trains in multiple, at times contradicting respects: capacity, energy conservation and stopping accuracy.

On one side, the essential task of TMS is to generate a detailed, global, conflict-free schedule for a given set of train lines and frequencies. The representation of conflict-free schedule is the production plan as the input to the ATO, which contains information as track path, train start time, travel time, and train speed profile, etc. Laube and Schaffer [13] introduced a decomposition of the railway network into condensation and compensation zones. Condensation zones lie in the proximity of main stations, where capacity is limited and the strategy is to travel with maximum speed. While in the compensation zone, which connect the condensation zones, its traffic is less dense and hence strategies supporting energy conservation or speed profile optimization could be introduced. This means that each TMS zone is dealt with according to its specific properties, resulting in a specific production plan for the train depending on the zone. This newly proposed framework with the approach described above will be applied in the TMS scheduling algorithm design.

On the other side, ATO aims to help trains to execute the production plan precisely and efficiently. This research will adopt the so-called optimized ATO method to further improve the production plan and realize accurate implementation. Similar to TMS, optimized ATO also proposed a decomposition of train operation into constant speed zones and stopping zones. Constant speed zones focus on capacity and energy conservation performance, whereas stopping 


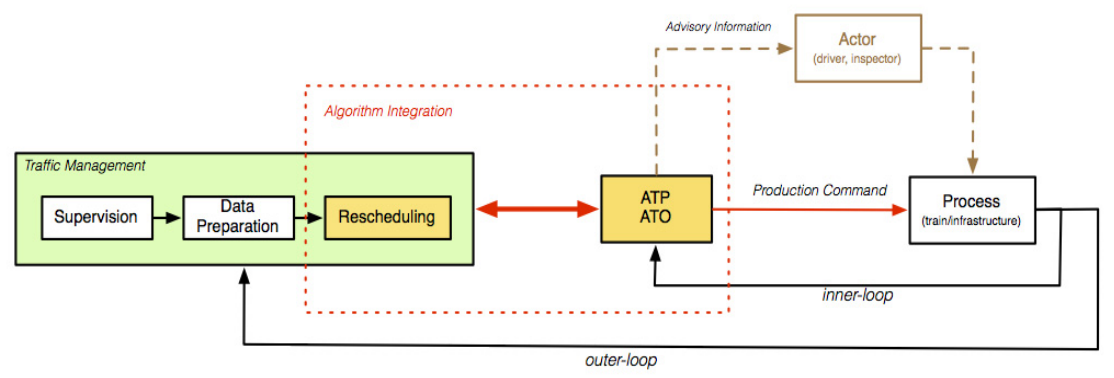

Figure 4: General control loop of overall optimization of train traffic.

zones emphasis the trains' stopping accuracy. The details of optimized ATO will be introduced in the next section.

\subsection{Optimized ATO}

\subsubsection{Introduction}

In general, the main task of ATO is to decide the best control command for the train at each time instant. The control commands range from powering to braking as discrete value. Different command contributes to different train speed. Therefore, as mentioned previously, the conventional ATO outputs commands to ensure train speed to follow a pattern target speed at each time instant. The typical control method applied is the Proportional Integral Derivative (PID), which only aims at minimizing the deviation between train speed and the target speed. But, except automatic following the target speed, Yasunobu et al. [14] proposed that ATO is able to improve more, such as energy conservation, accurate stopping, riding comfort, and so on. That is because ATO is located on the train, which has more knowledge of real-time states from the train. All of this knowledge gathered together enables optimized ATO to achieve further optimization on the train. Typical example is in Japan Shinkansen, where the resulting idea of multi-objectives optimization is implemented with the predictive fuzzy control. Compared to PID method, predictive fuzzy control results in better train performance, but it could be further improved in ATO framework and functional scalability by combination of predictive fuzzy control method with Analytical Hierarchy Process (AHP) method. The following subsections will provide detail description.

\subsubsection{Analysis of predictive fuzzy control in optimized ATO}

The predictive fuzzy control method is derived from the experience of excellent drivers. In this method, the driver's control knowledge is converted into a computer algorithm. By using fuzzy logic control, the control rule that represents the prediction of the future state gained from the experience of driver is valuated and the best control command is selected [14]. A general process of this method is described as follows. 
First, to follow the driver's strategy, the train operation process is decomposed into two parts. One is the called as Constant Speed Control (CSC), and another is Train Automatic Stop Control (TASC), whereas the TASC is a fixed area for train to execute accurate stopping.

Second, according to the fuzzy language, the drivers' verbal experience could be converted into fuzzy control rule as follow:

$$
R_{i} \text { : if }\left(\mathrm{u} \text { is } \mathrm{C}_{i} \rightarrow \mathrm{x} \text { is } \mathrm{A}_{i} \text { and } \mathrm{y} \text { is } \mathrm{B}_{i}\right) \text { then } \mathrm{u} \text { is } \mathrm{C}_{i} \text {. }
$$

In eqn (1), $\mathrm{u}$ is the control command, $\mathrm{x}$ and $\mathrm{y}$ are evaluation indices, $\mathrm{A}$ and $\mathrm{B}$ are fuzzy subsets that define different control objectives separately. $\mathrm{C}$ is the set of all the control commands. Therefore this fuzzy rule means if this command $u$ satisfies the objective A and B in the same time, then this command $u$ is selected.

Yasunobu et al. [14] depicts a clear perspective of evaluation of fuzzy rule by max-min composition of fuzzy relations. These predictive results are defined by the following fuzzy set $X\left(C_{i}, t\right)$ and $Y\left(C_{i}, t\right)$ with the membership function $\mu_{x C i t}(x)$ and $\mu_{y C i t}(y)$. At time $\mathrm{t}, P_{i \mid t}$ shows fuzzy rule assumption part as:

$$
P_{i \mid t}=\left(A_{i} \cap X\left(C_{i}, \mathrm{t}\right)\right) \times\left(\mathrm{B}_{i} \cap Y\left(C_{i}, t\right)\right) .
$$

Maximum value $r_{i}(t)$ of $P_{i \mid t}$ at time t is calculated from

$$
r_{i}(t)=\left.\sup _{x, y \in V \times U} \mu_{P i}\left(C_{i}: x, y\right)\right|_{t} .
$$

Lastly, the evaluation result $\mathrm{r}(\mathrm{t})$ of the whole control rules at time $\mathrm{t}$ is decided by eqn (4)

$$
r(t)=\max _{i} r_{i}(t)
$$

Therefore the best control instruction $u(t)$ is proposed.

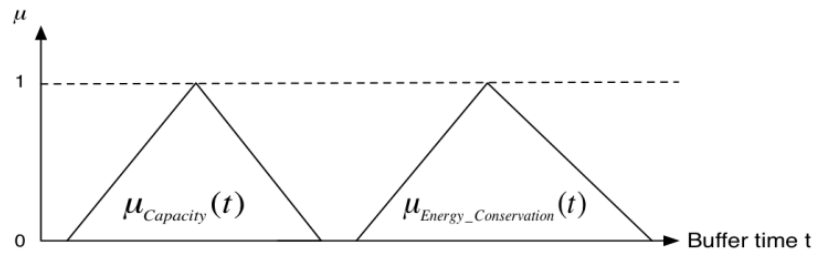

Figure 5: The case of fuzzy subsets based on one evaluation.

However, several control objectives (such as A or B in eqn (1) might be evaluated by the same index. Moreover, it is possible that there is no intersection between two different fuzzy subsets. For example in Figure 5, take the train running buffer time as an evaluation index, which could evaluate capacity as well as energy conservation. The two fuzzy sets of capacity and energy conservation might have no intersection based on the index of buffer time. That is because less buffer-time indicates increasing capacity; and this, in turn, leads to certain adverse effects on adopting coasting or cruising to reduce energy 
consumption [10]. In this article, it is called as the "objective-conflict problem". When this case happens, the max-min composition of fuzzy relations does not work. Besides, there are only two evaluation factors in the fuzzy rule assumption. But in fact, in order to further improve the train performance, there might be more evaluation factors to be considered. Due to these reasons, the AHP method will be introduced in the next part as a complement of predictive fuzzy control method to achieve better ATO performance.

\subsubsection{Integration of AHP and predictive fuzzy control in optimized ATO}

As mentioned previously, the core of ATO is a decision-making problem. What we need is not a more complicated way of thinking but a framework that will enable us to think of complex problems in a simple way [15]. The AHP method could be helpful for improving optimized ATO, which combines system approach and deductive approach into an integrated, logical framework.

The system thinking is addressed by structuring ideas hierarchically, that Figure 6 gives an example [10]. This structure contains three levels. Top level is the overall objective; middle levels are criteria as control objectives contributed to the goal; lowest level is alternatives as system solutions. In the ATO example, the overall goal is to decide the best control command at each time instant. The criteria are derived from driver's experience as previous predictive fuzzy control method. The solution level supposes 16 different control commands range from maximum traction $\mathrm{P} 7$ to cruising command $\mathrm{P}$, from maximum braking $\mathrm{B} 7$ to minimum braking $\mathrm{B} 1$, and coasting command. Compared to predictive fuzzy control, AHP structure delivers a clear and logic structure to describe the system as well as flexible to accommodate new criteria.

The causal thinking or explanation of criteria relationship is developed through paired comparison of elements in the hierarchy. It is to establish priorities for each criterion of the hierarchy, synthesize judgments to yield a set

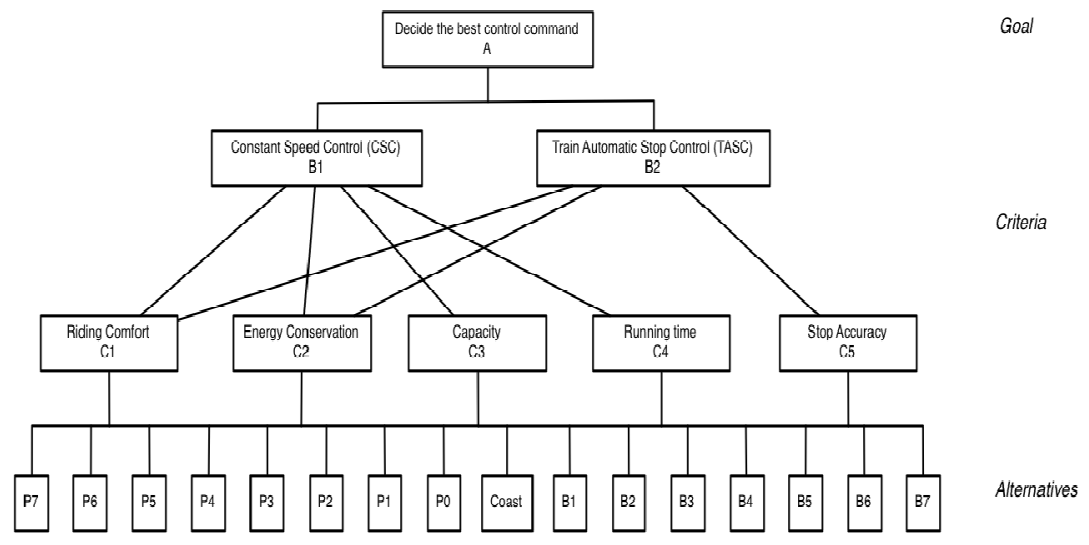

Figure 6: Analytical hierarchy structure applied in optimized ATO [10]. 
of overall priorities, check the consistency of these judgements and combine to a final decision based on the results of this process [15].

A matrix is the preferred form to do the pairwise comparisons, that is, to compare the criterion in pairs against a given criterion. To fill in the matrix of pairwise comparisons, different numbers are used to represent the relative importance of one criterion over another with respect to the property. The numbers as fundamental scale for pairwise comparisons are defined in the Table 1.

Table 1: $\quad$ The fundamental scale for pairwise comparisons [15].

\begin{tabular}{|c|c|c|}
\hline $\begin{array}{c}\text { Intensity of } \\
\text { Importance }\end{array}$ & Definition & Explanation \\
\hline 1 & Equal importance & Two activities contribute equally to the objective. \\
\hline 3 & Moderate importance & $\begin{array}{c}\text { Experience and judgment slightly favour one } \\
\text { activity over another. }\end{array}$ \\
\hline 5 & Strong importance & $\begin{array}{c}\text { Experience and judgment strongly favour one } \\
\text { activity over another. }\end{array}$ \\
\hline 7 & $\begin{array}{c}\text { Very strong or demonstrated } \\
\text { importance }\end{array}$ & $\begin{array}{c}\text { An activity is favoured very strongly over } \\
\text { another; its dominance demonstrated in practice. }\end{array}$ \\
\hline $2,4,6,8$ & $\begin{array}{c}\text { Extreme importance } \\
\text { For compromise between the } \\
\text { above values } \\
\text { is of the highest possible order of affirmation. }\end{array}$ & $\begin{array}{c}\text { Sometimes one needs to interpolate a } \\
\text { compromise judgment numerically because there } \\
\text { is no good word to describe it. }\end{array}$ \\
\hline $\begin{array}{c}\text { Reciprocals } \\
\text { of above }\end{array}$ & $\begin{array}{c}\text { If activity } i \text { has one of the } \\
\text { above nonzero numbers } \\
\text { assigned to it when compared } \\
\text { with activity } j \text { then } j \text { has the } \\
\text { reciprocal value when } \\
\text { compared with } i\end{array}$ & $\begin{array}{c}\text { A comparison mandated by choosing the smaller } \\
\text { element as the unit to estimate the larger one as } \\
\text { multiple of that unit. }\end{array}$ \\
\hline
\end{tabular}

Based on this scale, pairwise comparison in criteria $\mathrm{B}$ and $\mathrm{C}$ are shown in Figure 7. Each priority is calculated using the following formula eqn (5) whereas eqn (6) and eqn (7) give examples of priority calculation in Figure 8. The final priorities for each objective in criteria $\mathrm{C}$ is shown in Figure 9. The step of pairwise comparison is to resolve the "objective-conflict" problem by calculating priority with respect to each control objective in criteria C. It is a subjective method that reflects human's thinking and experience.

$$
\begin{gathered}
\text { Priority } \left._{k}=\frac{1}{n} \sum_{j=1}^{n} \frac{\text { element }_{k j}}{\sum_{i=1}^{n} \text { element }_{i j}} \text { ( } i \text { is row number, } j \text { is column number }\right) . \\
w_{1}^{(2)}=\frac{1}{2}\left(\frac{1}{1+1 / 2}+\frac{2}{1+2}\right)=\frac{2}{3} \approx 0.67 . \\
w_{2}^{(2)}=\frac{1}{2}\left(\frac{1 / 2}{1+1 / 2}+\frac{1}{1+2}\right)=\frac{1}{3} \approx 0.33 .
\end{gathered}
$$

Normally, the next step is to develop five matrices for comparing 16 alternative solutions with respect to each criterion in C. But, instead of matrix comparison, fuzzy model calculation is adopted for each solution with respect to 


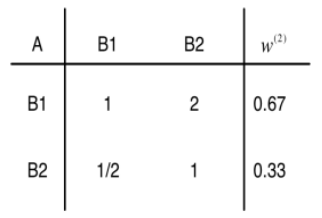

$\lambda_{\max }=2, C I=0$

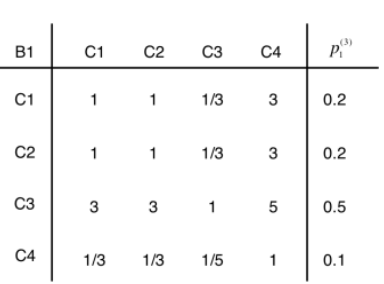

$\lambda_{\operatorname{mix}}=4.125, C I=4 \%$

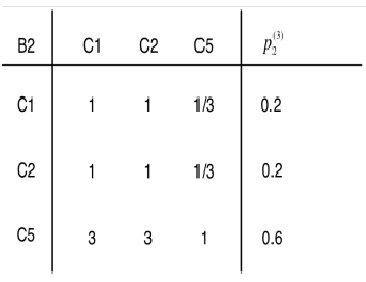

$\lambda_{\operatorname{mix}}=3, C I=0$

Figure 7: $\quad$ Criteria pairwise comparison [10].

\begin{tabular}{|c|c|c|c|}
\hline \multirow{2}{*}{$C^{B}$} & $w_{1}^{1} \cdot 1-0.07$ & $w_{2}:=0.33$ & \multirow{2}{*}{ 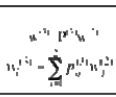 } \\
\hline & $P_{1}^{\left({ }^{(z)}\right)}$ & $P_{2}^{(3)}$ & \\
\hline $\mathrm{Cl}$ & 0.2 & 0.2 & 0.2 \\
\hline $\mathrm{C} 2$ & 0.2 & 0.2 & 0.2 \\
\hline $\mathrm{C} 3$ & 0.5 & 0 & 0.335 \\
\hline $\mathrm{C} 4$ & 0.1 & 0 & 0.067 \\
\hline C5 & 0 & 0.6 & 0.198 \\
\hline
\end{tabular}

Figure 8: $\quad$ Priorities for each control objective [10].

\begin{tabular}{|c|c|c|c|c|c|c|}
\hline & $\begin{array}{r}\mathrm{C} 1 \\
(0.2) \\
\end{array}$ & $\begin{array}{l}\mathrm{C} 2 \\
(0.2) \\
\end{array}$ & $\begin{array}{c}\text { C3 } \\
(0.335) \\
\end{array}$ & $\begin{array}{c}\text { C4 } \\
(0.067) \\
\end{array}$ & $\begin{array}{c}\text { C5 } \\
(0.198) \\
\end{array}$ & $\begin{array}{c}\text { Vector of Overall } \\
\text { Priorities }\end{array}$ \\
\hline $\mathrm{P} 7$ & $\mu_{C \perp \mid \mathrm{P} 7}(0.2)$ & $+\mu_{C 2 \mid \mathrm{P} 7}(0.2)$ & $+\mu_{C 3 \mid \mathrm{PT}}(0.335)$ & $+\mu_{C 4 \mid \mathrm{PT}}(0.067)$ & $+\mu_{C S \mid P 7}(0.198)$ & $w_{1}$ \\
\hline$\cdots$ & & & $\cdots$ & & & $\cdots$ \\
\hline $\mathrm{PO}$ & $\mu_{C 11 \mathrm{PO}}(0.2)$ & $+\mu_{C 21 \mathrm{PO}}(0.2)$ & $+\mu_{c 3 \mid 10}(0.335)$ & $+\mu_{C 4 \mid \mathrm{PO}}(0.067)$ & $+\mu_{c S \mid \mathrm{PO}}(0.198)$ & $w_{8}$ \\
\hline Coast & $\mu_{c 1 \text { coses }}(0.2)$ & $+\mu_{c 2 \text { lcase }}(0.2)$ & $+\mu_{c 31 \text { coser }}(0.335)$ & $+\mu_{c 41 \text { const }}(0.067)$ & $+\mu_{c 5 \text { loost }}(0.198)$ & $w_{9}$ \\
\hline $\mathrm{B} 1$ & $\mu_{C \mid \mathrm{BB}}(0.2)$ & $+\mu_{c 2 \mid \mathrm{B} 1}(0.2)$ & $+\mu_{C 3 \mid \mathrm{BI}}(0.335)$ & $+\mu_{C 4|\mathrm{~B}|}(0.067)$ & $+\mu_{c s \mid \mathrm{Bl}}(0.198)$ & $w_{10}$ \\
\hline$\cdots$ & & & $\cdots$ & & & $\cdots$ \\
\hline B7 & $\mu_{C 11 \mathrm{~B} 7}(0.2)$ & $+\mu_{C 2 \mid \mathrm{B} 7}(0.2)$ & $+\mu_{c 31 \mathrm{~B} 7}(0.335)$ & $+\mu_{C 4 \mid \mathrm{B} 7}(0.067)$ & $+\mu_{c 5 \mid \mathrm{B} 7}(0.198)$ & $w_{16}$ \\
\hline
\end{tabular}

Figure 9: Determining the overall priorities (Rao [10]).

each criterion $\mathrm{C}$. This is because the fuzzy model is more manageable and flexible to represent the locomotive relation between each criterion and alternative solutions. Specifically, each alternative solution results the specific effort exerted on the train, the effort indicates the value of accelerates. The indicated accelerate value enables to predict the speed or distance or running time value in the next time instant. In turn, the predictive speed or distance or running time information will evaluate the criterion performance with respect to each fuzzy model. For example in where $\mu_{\mathrm{c} 3 \mid \mathrm{P} 7}$ means the fuzzy result of capacity performance with the maximum powering command $\mathrm{P} 7, \mu_{\mathrm{c} 2[\mathrm{~B} 7}$ is the fuzzy result 
of energy conservation performance with maximum braking command B7. In the end, illustrates the final step to calculate the overall priorities for each solution at each time instant. As a result, the best solution is the command with the highest priority. In addition, the fuzzy model in this step could be the cooperation part with TMS. For instance, the TMS's production plan is essential to be considered in building the capacity fuzzy model as well as energy conservation fuzzy model.

\subsubsection{Implementation of optimized ATO}

Until now, the optimized ATO method mentioned previously is only applied in the scientific project of Southwest Jiaotong University in China. This scientific project is a simulation system of Chinese Railway Control System Level 2 and Level 3 (CTCS2 and CTCS3) where optimized ATO is an optional function for the On Board Unit (OBU) subsystem. The infrastructure data is getting from the Hefei-Nanjing rail line, whereas the train locomotive data are from high-speed train type CRH1, CRH2, CRH3 and CRH5. The whole optimized ATO simulation process is supervised by the ATP simulation system. It enables to choose different train type to run the simulation as well as performance comparison between the optimized ATO and human operation. The result showed that optimized ATO has obvious improvement in stopping accuracy and increased capacity except energy conservation. But this drawback is predicted to be fixed by the integration of optimized ATO with TMS in the future.

\section{Conclusion and future work}

This article aims at proposing the idea of integrating two existing separate optimization systems for infrastructure and for trains. It proposes to combine the rescheduling algorithm of TMS with multi-objective algorithm of optimized ATO, to improve railway performances as well as reduce railway cost. TMS strengths in the whole network scheduling while the optimized ATO does further improvement in accurate production plan implementation. Combining the strength of both TMS and optimized ATO will conceivably give insight on how to satisfy various optimization goals from the perspective of the infrastructure and the train.

In addition, this article proposes an integration algorithm for optimized ATO, to combine predictive fuzzy control method with the AHP method to achieve multi-objectives optimization. Based on previous control loop concept, this optimized ATO method will be applied into the research of holistic optimization approach in the inner feedback control loop.

The future work will be more concentrated on the TMS scheduling algorithm, to create a conflict-free railway schedule and realize rescheduling by predicting and preventing conflicts efficiently. After that it is essential to find a reasonable solution to combine the strength from both TMS and ATO. It will not only propose algorithm integration, but also deliver a model for evaluation, as well as a case study based on practical project. Now the whole project is undertaking in Systransis Ltd (which is mentioned previously as the company to develop AF 
system in Lötschberg Base Tunnel) as well as with the consulting help from the Institute for Transport Planning and Systems (IVT) in ETH. Both Systransis and IVT are sophisticated in TMS rescheduling algorithms that will be a great help to support this holistic optimization research.

\section{References}

[1] UIC, Compendium on ERTMS (European Rail Traffic Management System), DVV Media Group GmbH | Eurailpress, pp. 32, 2009.

[2] Massaki, H., Sakuma, H. and Nishijima, E., Recent train traffic management systems for public and private railways, Hitachi Review, Vol 53 (1), pp. 30-34, 2004.

[3] Hitachi Ltd., http://www.hitachi-rail.com/products/operation and_management/traffic_management_systems/features/index.html

[4] Telvent - Rail operation management systems, http://www.railwaytechnology.com/contractors/signal/telvent/

[5] Thales NetTrac 6613 ARAMIS, http://www.thalesgroup.com/ Portfolio/Transportation/NetTrac 6613 ARAMIS/?pid=14250

[6] Metha, F., Rößiger, C. and Montigel, M., Latent energy savings due to the innovative use of advisory speeds to avid occupation conflicts, Computers in Railways XII, Vol 114, 2010.

[7] Montigel, M., Operations control system in the Lötschberg Base Tunnel, RTR, Vol 2, 2009.

[8] Lüthi, M., Improving the efficiency of heavily used railway networks through integrated real-time rescheduling, Diss.ETH No.18615, pp. 54-55, 2009.

[9] Poré, J., ATO for suburban and main lines, IRSE-ITC, 2010.

[10] Rao, X., The research and simulation of automatic train operation algorithm, Southwest Jiaotong University Master Degree Thesis, 2007.

[11] Tang, T. and Huang, L., System analysis and design of automatic train operation on Metro, Journal of Northern Jiaotong University, Vol 26 (3), 2002.

[12] Yasui, Y., Automatic train operation system for the high speed Shinkansen train, Computers in Railways X, Vol 88, 2006.

[13] Laube, F. and Schaffer, H., Specific working results about strategic network division for the PULS 90 project, Working paper, SBB Bern, 2003.

[14] Yasunobu, S., Miyamoto, S. and Ihara, H., A fuzzy control for train automatic stop control, Trans. Of the Society of Instrument and Control Engineers, Vol E2, 2009.

[15] Thomas, S., Decision making for leaders: the analytic hierarchy process for decisions in a complex world, University of Pittsburgh, pp. 13-18, 7088, 1995. 